University of Wollongong

Research Online

Australian Institute for Innovative Materials -

Papers

Australian Institute for Innovative Materials

$1-1-2019$

\title{
Engineering additional edge sites on molybdenum dichalcogenides toward accelerated alkaline hydrogen evolution kinetics
}

Qian Zhou

University of Wollongong, qz704@uowmail.edu.au

Guoqiang Zhao

University of Wollongong, gz815@uowmail.edu.au

Kun Rui

University of Wollongong, krui@uow.edu.au

Yaping Chen

University of Wollongong, yc463@uowmail.edu.au

Xun Xu

University of Wollongong, xun@uow.edu.au

See next page for additional authors

Follow this and additional works at: https://ro.uow.edu.au/aiimpapers

Part of the Engineering Commons, and the Physical Sciences and Mathematics Commons

Research Online is the open access institutional repository for the University of Wollongong. For further information contact the UOW Library: research-pubs@uow.edu.au 


\title{
Engineering additional edge sites on molybdenum dichalcogenides toward accelerated alkaline hydrogen evolution kinetics
}

\begin{abstract}
The sluggish reaction kinetics of the hydrogen evolution reaction (HER) in alkaline media is a great obstacle to alkaline water electrolysis, and it remains a great challenge to develop precious metal-free efficient catalysts for the alkaline HER. Transition metal dichalcogenides (TMDs), in particular MoS2 and MoSe2, are promising catalysts for the HER in acidic media, but they exhibit much inferior catalytic activity for the alkaline HER owing to the slow water dissociation process. In this work, we, for the first time, demonstrate that TMD heterostructures with abundant edge sites deliver substantially accelerated alkaline HER kinetics, which is in great part due to the enhanced water adsorption/dissociation capability. As a proof of concept, MoS2/MoSe2 heterostructures with ultrasmall MoS2 nanoclusters anchored on MoSe2 nanosheets are synthesized via a solution-phase process and are investigated as alkaline HER catalysts in detail. MoSe2 nanosheets serve as excellent substrates to hinder the agglomeration of MoS2 nanoclusters, resulting in abundant edge sites. Benefiting from the decent water adsorption/dissociation capability of the edge sites, the optimal MoS2/MoSe2 heterostructure shows exceptional catalytic activity in $1 \mathrm{M} \mathrm{KOH}$ with an overpotential of $235 \mathrm{mV}$ at $10 \mathrm{~mA} \mathrm{~cm}-2$ and a Tafel slope of $96 \mathrm{mV} \mathrm{dec}-1$, which is substantially improved as compared with the individual MoSe2 (330 mV, $135 \mathrm{mV}$ dec-1) and MoS2 (400 $\mathrm{mV}, 157 \mathrm{mV}$ dec-1). The success of this catalyst design strategy for enhancing alkaline HER kinetics is also demonstrated in MoSe2/MoSe2 and MoS2/MoS2 heterostructures. The results suggest that engineering additional edge sites that have a strong affinity for $\mathrm{H} 2 \mathrm{O}$ is critical for TMDs towards enhanced alkaline HER activity, and also open new avenues in the design of precious metal-free efficient catalysts for the alkaline HER.

Disciplines

Engineering | Physical Sciences and Mathematics

\section{Publication Details}

Zhou, Q., Zhao, G., Rui, K., Chen, Y., Xu, X., Dou, S. Xue. \& Sun, W. (2019). Engineering additional edge sites on molybdenum dichalcogenides toward accelerated alkaline hydrogen evolution kinetics. Nanoscale, 11 (2), 717-724.
\end{abstract}

\section{Authors}

Qian Zhou, Guoqiang Zhao, Kun Rui, Yaping Chen, Xun Xu, Shi Xue Dou, and Wenping Sun 


\section{Engineering Additional Edge Sites on Molybdenum Dichalcogenides toward}

\section{Accelerated Alkaline Hydrogen Evolution Kinetics}

Qian Zhou ${ }^{+}$, Guoqiang Zhao ${ }^{+}$, Kun Rui, Yaping Chen, Xun Xu* , Shi Xue Dou, Wenping Sun* Institute for Superconducting and Electronic Materials, Australian Institute for Innovative Materials, University of Wollongong, Wollongong, NSW 2522, Australia

\section{Abstract}

The sluggish reaction kinetics of hydrogen evolution reaction (HER) in alkaline media is a great obstacle to alkaline water electrolysis, and it remains a great challenge to develop precious metal-free efficient catalysts for alkaline HER. Transition metal dichalcogenides (TMDs), in particular $\mathrm{MoS}_{2}$ and $\mathrm{MoSe}_{2}$, are promising catalysts for HER in acidic media, but they exhibit much inferior catalytic activity for alkaline HER owing to the slow water dissociation process. In this work, we, for the first time, demonstrate that TMDs heterostructures with abundant edge sites deliver substantially accelerated alkaline HER kinetics, which is in great part due to the enhanced water adsorption/dissociation capability. As a proof of concept, $\mathrm{MoS}_{2} / \mathrm{MoSe}_{2}$ heterostructures with ultrasmall $\mathrm{MoS}_{2}$ nanoclusters anchored on $\mathrm{MoSe}_{2}$ nanosheets are synthesized via a solution-phase process and are investigated as alkaline HER catalysts in detail. $\mathrm{MoSe}_{2}$ nanosheets serve as excellent substrates to hinder the agglomeration of $\mathrm{MoS}_{2}$ nanoclusters, resulting in abundant edge sites. Benefiting from the decent water adsorption/dissociation capability of the edge sites, the optimal $\mathrm{MoS}_{2} / \mathrm{MoSe}_{2}$ heterostructure shows exceptional catalytic activity in $1 \mathrm{M} \mathrm{KOH}$ with an overpotential of $235 \mathrm{mV}$ at $10 \mathrm{~mA} \mathrm{~cm}^{-2}$ and a Tafel slope of $96 \mathrm{mV} \mathrm{dec}{ }^{-1}$, which is substantially improved as compared with the individual $\mathrm{MoSe}_{2}\left(330 \mathrm{mV}, 135 \mathrm{mV} \mathrm{dec}{ }^{-1}\right)$ and $\mathrm{MoS}_{2}\left(400 \mathrm{mV}, 157 \mathrm{mV} \mathrm{dec}{ }^{-1}\right)$. The success of this catalyst design strategy for enhancing

* Corresponding author.

E-mail address: xun@uow.edu.au (X.Xu), wenping@uow.edu.au (W. Sun)

${ }^{+}$Q. Zhou and G. Zhao contributed equally to this work. 
alkaline HER kinetics is also demonstrated in $\mathrm{MoSe}_{2} / \mathrm{MoSe}_{2}$ and $\mathrm{MoS}_{2} / \mathrm{MoS}_{2}$ heterostructures. The results suggest that engineering additional edge sites that have a strong affinity for $\mathrm{H}_{2} \mathrm{O}$ is critical for TMDs towards enhanced alkaline HER activity, and also open new avenues in the design of precious metal-free efficient catalysts for alkaline HER.

Keywords: heterostructure, edge site, molybdenum dichalcogenide, hydrogen evolution reaction, alkaline water electrolysis

\section{Introduction}

The energy crisis and environmental pollution problems have prompted the research for clean energy carrier. ${ }^{1-4}$ Hydrogen has attracted significant attention owing to the zero greenhouse gas emission as well as high gravimetric energy density. 5,6 Among various hydrogen generation strategies, water electrolysis, particularly driven by renewable energies, has already demonstrated its feasibility in industrial processes. ${ }^{7-9}$ However, developing efficient and earth-abundant alternatives to noble metals which are the most satisfying electrocatalysts, are still the center for realizing the large-scale application of hydrogen generation via water electrolysis. $^{10,11}$ Practically, water electrolysis is more favoured in alkaline media which helps to promote the sluggish oxygen evolution reaction (OER) to achieve a high energy conversion efficiency. ${ }^{12-15}$ However, most earth-abundant electrocatalysts such as transition metal chalcogenides (TMDs), ${ }^{16-20}$ nitrides, ${ }^{21,22}$ carbides $^{23,24}$ and phosphides, ${ }^{25-28}$ despite of the performance comparable to noble metal that they may possess in acid media, exhibit inferior hydrogen evolution reaction (HER) activity in alkaline media due to the sluggish water adsorption/dissociation step. ${ }^{29-31}$

Molybdenum dichalcogenides $\left(\mathrm{MoS}_{2}\right.$ and $\left.\mathrm{MoSe}_{2}\right)$, as typical TMDs, are among the most efficient catalysts for HER in acid media owing to the moderate Gibbs free energy for hydrogen adsorption. ${ }^{32,} 33$ Both theoretical calculations and experimental results have demonstrated that unsaturated $\mathrm{Mo}-\mathrm{S}$ sites possess the optimal hydrogen adsorption free 
energy, ${ }^{34-37}$ which have stimulated the development of nanostructured TMDs-based catalysts with enriched edge sites. ${ }^{38,39}$ As for alkaline HER, a recent theoretical study demonstrated that the water adsorption/dissociation process is more favoured on the edge sites rather than the basal plane of $\mathrm{MoS}_{2}{ }^{40}$ Thus, increasing the exposure of TMDs edge sites might also be an effective strategy for promoting alkaline hydrogen evolution kinetics. Herein, as a proof of concept, a series of TMDs-based heterostructures $\left(\mathrm{MoS}_{2} / \mathrm{MoSe}_{2}, \mathrm{MoSe}_{2} / \mathrm{MoSe}_{2}\right.$, and $\mathrm{MoS}_{2} / \mathrm{MoS}_{2}$ ) with $\mathrm{MoS}_{2}$ or $\mathrm{MoSe}_{2}$ quantum dots decorated on the basal planes of TMDs nanosheets were synthesized via a two-step chemical solution process. The $\mathrm{MoS}_{2}$ or $\mathrm{MoSe}_{2}$ quantum dots provide abundant edge sites which substantially enhance the affinity for $\mathrm{H}_{2} \mathrm{O}$, and the TMDs-based heterostructures exhibit significantly improved catalytic activities over the individual nanosheets in alkaline media. The results not only demonstrate the feasibility of engineering additional edges sites on molybdenum dichalcogenides toward the development of efficient alkaline HER electrocatalysts but also provide new insights into indepth understanding of the HER mechanism.

\section{Experiment section}

\subsection{Materials synthesis}

Synthesis of $\mathrm{MoSe}_{2}$ nanosheets: $\mathrm{MoSe}_{2}$ nanosheets were synthesized by a hydrothermal method. Briefly, $2 \mathrm{mmol}$ Se powder and $1 \mathrm{mmol} \mathrm{Na} \mathrm{MoO}_{4} \cdot 2 \mathrm{H}_{2} \mathrm{O}$ were dispersed in $30 \mathrm{~mL}$ deionized water (DI-W) under stirring at room temperature to form a dark red-brown suspension. Then, $2.5 \mathrm{mmol} \mathrm{NaBH}_{4}$ was slowly added into the above suspension and dissolved completely. Subsequently, the mixture was transferred into a $50 \mathrm{~mL}$ Teflon stainless-steel autoclave with Ar purging for $30 \mathrm{~min}$, and then the autoclave was heated at $180{ }^{\circ} \mathrm{C}$ for $24 \mathrm{~h}$ in an electric oven. After the reaction was completed, black products were 
collected by centrifugation, washed with DI-W and ethanol three times, and eventually dried overnight in a vacuum oven at $60{ }^{\circ} \mathrm{C}$.

Synthesis of $\mathrm{MoS}_{2} / \mathrm{MoSe}_{2}$ nanosheets: Typically, $0.1 \mathrm{mmol} \mathrm{MoSe} \mathrm{M}_{2}$ was dispersed in $35 \mathrm{~mL}$ DI-W and treated by ultrasonication for $3 \mathrm{~h}$ to form a homogeneous suspension. Then various amount of $\mathrm{Na}_{2} \mathrm{MoO}_{4} \cdot 2 \mathrm{H}_{2} \mathrm{O}(0.025,0.05$ and $0.1 \mathrm{mmol})$ and L-cysteine $(0.1,0.2$ and $0.4 \mathrm{mmol})$ dissolved in above suspension under stirring. Afterwards, the mixture was transferred into a $50 \mathrm{~mL}$ Teflon-lined stainless steel autoclave and was heated at $180{ }^{\circ} \mathrm{C}$ for $36 \mathrm{~h}$. The products were collected by centrifugation, washed with DI-W and ethanol three times, and eventually dried overnight in a vacuum oven at $60{ }^{\circ} \mathrm{C}$. Based on the molar ratio of $\mathrm{MoS}_{2}$ to $\mathrm{MoSe}_{2}$, the as-obtained hybrids are denoted as $\mathrm{MoS}_{2} / \mathrm{MoSe}_{2}-0.25, \mathrm{MoS}_{2} / \mathrm{MoSe}_{2}-0.5$, and $\mathrm{MoS}_{2} / \mathrm{MoSe}_{2}-1$, respectively.

Synthesis of $\mathrm{MoS}_{2}$ nanosheets: The procedures for synthesizing $\mathrm{MoS}_{2}$ nanosheets were the same as that of $\mathrm{MoS}_{2} / \mathrm{MoSe}_{2}$ nanosheets except that no $\mathrm{MoSe}_{2}$ sheets were added.

Synthesis of $\mathrm{MoSe}_{2} / \mathrm{MoSe}_{2}$ nanosheets: A certain amount of $\mathrm{MoSe}_{2}$ nanosheets was added into $35 \mathrm{~mL}$ water and treated with ultrasonication for $3 \mathrm{~h}$ to form a suspension. Then the following steps are the same as that of synthesising pure $\mathrm{MoSe}_{2}$ nanosheets.

Synthesis of $\mathrm{MoS}_{2} / \mathrm{MoS}_{2}$ nanosheets: A certain amount of $\mathrm{MoS}_{2}$ nanosheets was added into 35 $\mathrm{mL}$ water and treated with ultrasonication for $3 \mathrm{~h}$ to form a suspension. And the following steps are the same as that of synthesis of pure $\mathrm{MoS}_{2}$ nanosheets.

\subsection{Physical Characterization}

The morphology and crystal structure of the as-prepared products were investigated using a JEM-2010 transmission electron microscope (TEM) operated at $200 \mathrm{kV}$. Scanning transmission electron microscopy (STEM) images were acquired on a probe-corrected JEOL 
ARM200F TEM operated at $200 \mathrm{kV}$. Energy disperse X-ray (EDS) mapping images were acquired on FEI Talos F200X equipped with four symmetrical EDS signal detectors, operated at $200 \mathrm{kV}$. The powder X-ray diffraction (XRD) patterns were recorded on a GBC Scientific Equipment LLC X-ray powder diffractometer with $\mathrm{Cu} \mathrm{K} \alpha$ radiation $(\lambda=1.541 \AA, 25 \mathrm{~mA}, 40$ $\mathrm{kV}, 2{ }^{\circ} \mathrm{min}^{-1}$ ). The X-ray photoelectron spectroscopy (XPS) spectra of samples were performed on an XPS, Thermo ESCALAB 250Xi instrument with Al $\mathrm{K} \alpha$ as the excitation source.

\subsection{Electrochemical measurements}

All the electrochemical experiments were conducted with a rotating disc electrode (RDE) systems using a three-electrode system at room temperature, where the electrochemical catalysts were used as the working electrodes, platinum wire as the counter electrode, and $\mathrm{Hg} / \mathrm{HgO}$ and $\mathrm{Ag} / \mathrm{AgCl}$ as the reference electrodes in $1.0 \mathrm{M} \mathrm{KOH}$ and $0.5 \mathrm{M} \mathrm{H}_{2} \mathrm{SO}_{4}$, respectively. The experiments were controlled by a WaveDriver 20 electrochemistry workstation (Pine Research Instruments, US). The working electrode was prepared via the method reported in our previous work. ${ }^{41}$ In brief, the as-synthesized catalyst $(4 \mathrm{mg})$ was first added in $1 \mathrm{~mL}$ water-isopropanol mixed solution (volume ratio of $3: 1$ ) containing $30 \mu \mathrm{L}$ Nafion solution (5 wt\%) and treated with ultrasonication to form a homogeneous ink. Then $10 \mu \mathrm{L}$ catalyst ink was drop-cast onto a glassy carbon electrode (GCE, $5 \mathrm{~mm}$ in diameter) and dried naturally (catalyst mass loading: $0.204 \mathrm{mg} \mathrm{cm}^{-2}$ ). LSV curves were obtained at a scan rate of $5 \mathrm{mV} \mathrm{s}^{-1}$ at the rotation speed of $1600 \mathrm{rpm}$, and the results were corrected with $90 \%$ iR-compensation. All potentials were calibrated and reported with respect to reverse hydrogen electrode (RHE) by the equations: $\mathrm{E}(\mathrm{RHE})=\mathrm{E}(\mathrm{Hg} / \mathrm{HgO})+0.924 \quad \mathrm{~V}$ and $\mathrm{E}(\mathrm{RHE})=\mathrm{E}(\mathrm{Ag} / \mathrm{AgCl})+0.197 \mathrm{~V}$. EIS measurements were carried out in the frequency range of $100 \mathrm{kHz}$ to $100 \mathrm{mHz}$ at potential of $-0.3 \mathrm{~V}$ versus RHE. The turnover frequency is calculated using the following equations: ${ }^{42}$ 


$$
\text { TOF }=\frac{\text { Total number of } \mathrm{H}_{2} \text { atoms per second }}{\text { Total number of active sites per unit area }}=\frac{\frac{j}{2 \times q}}{\mathrm{~N}}
$$

Where $\mathrm{j}$ is the current density $\left(\mathrm{A} \mathrm{cm}^{-2}\right)$ at a given overpotential, $\mathrm{q}$ is the elementary charge $\left(1.6 \times 10^{-19} \mathrm{C}\right), \mathrm{N}$ is the number of surface Mo atom per unit area which is estimated to be $1.06 \times 10^{15} \mathrm{~cm}^{-2}$ and $1.15 \times 10^{15} \mathrm{~cm}^{-2}$ for $\mathrm{MoSe}_{2}$ and $\mathrm{MoS}_{2}$, respectively.

\section{Results and discussion}

Fig. 1a shows the schematic representation for the growth of 2D $\mathrm{MoS}_{2} / \mathrm{MoSe}_{2}$ vertical heterostructure. L-cysteine and $\mathrm{MoO}_{4}{ }^{2-}$ firstly absorb on the surface of $\mathrm{MoSe}_{2}$ nanosheets, and then $\mathrm{MoO}_{4}{ }^{2-}$ is reduced to $\mathrm{MoS}_{2}$ by L-cysteine during the hydrothermal process, during which process $\mathrm{MoSe}_{2}$ nanosheets play an important role in the confined growth of $\mathrm{MoS}_{2}$ quantum dots. As depicted in Fig. S1 (supporting information), pure $\mathrm{MoSe}_{2}, \mathrm{MoS}_{2}$, and $\mathrm{MoS}_{2} / \mathrm{MoSe}_{2}$ heterostructures show similar X-ray diffraction (XRD) patterns, in which peaks at around $32.0^{\circ}, 38.3^{\circ}$, and $56.4^{\circ}$ can be assigned to (100), (103), and (110) planes of molybdenum dichalcogenides. It is worth noting that the (002) peak of the pure $\mathrm{MoSe}_{2}$, unlike $\mathrm{MoS}_{2}$ and $\mathrm{MoS}_{2} / \mathrm{MoSe}_{2}$, cannot be observed in the XRD patterns owing to the ultrathin thickness of $\mathrm{MoSe}_{2}$ nanosheets. ${ }^{43}$ The typical morphology of pristine $\mathrm{MoSe}_{2}$ nanosheets is shown in Fig. S2a and b (Supporting Information), and the lattice fringes can be well assigned to $\mathrm{MoSe}_{2}$ (Fig. S2c and d). Taking $\mathrm{MoS}_{2} / \mathrm{MoSe}_{2}-0.5$ as an example, as shown in Fig. $1 b$ and Fig. S3a and b (Supporting Information), the $\mathrm{MoS}_{2} / \mathrm{MoSe}_{2}-0.5$ heterostructure inherits similar nanosheets morphology as $\mathrm{MoSe}_{2}$. The specific surface areas of $\mathrm{MoSe}_{2}$ and $\mathrm{MoS}_{2}-\mathrm{MoSe}_{2}-0.5$ are determined to be 48 and $61 \mathrm{~m}^{2} / \mathrm{g}$ by BET (Fig. S3c, Supporting Information), respectively. The lattice fringe with spacing of $0.68 \mathrm{~nm}$ shown in Fig. $1 \mathrm{~b}$ corresponds to the typical (002) planes of $\mathrm{MoSe}_{2}$ substrates. However, it is difficult to distinguish $\mathrm{MoS}_{2}$ species from $\mathrm{MoSe}_{2}$ substrates because of the same crystal structure and 
similar crystal parameters of $\mathrm{MoS}_{2}$ and $\mathrm{MoSe}_{2}$ in the high-resolution TEM (HRTEM) image (Fig. 1c). However, two sets of diffraction spots are shown in the corresponding fast Fourier transformation (FFT) patterns (Fig. 1d). Specifically, a set of six spots with a lattice spacing of about $0.27 \mathrm{~nm}$ can be ascribed to the $\{100\}$ planes of $\mathrm{MoS}_{2}$, and the other set with a lattice spacing of $0.28 \mathrm{~nm}$ can be assigned to $\{100\}$ planes of $\mathrm{MoSe}_{2}$. Two patterns are regenerated by applying inversed FFT (IFFT) on the selected FFT patterns (Fig. 1e), which show clear boundaries between $\mathrm{MoS}_{2}$ and $\mathrm{MoSe}_{2}$ as well as the ultrasmall size of $\mathrm{MoS}_{2}$ quantum dots $(<5 \mathrm{~nm})$ on the $\mathrm{MoSe}_{2}$ nanosheets. Both $\mathrm{MoS}_{2} / \mathrm{MoSe}_{2}-0.25$ and $\mathrm{MoS}_{2} / \mathrm{MoSe}_{2}-1$ show similar morphology to $\mathrm{MoS}_{2} / \mathrm{MoSe}_{2}-0.5$ heterostructure, as can be seen in Fig. S4 (Supporting Information). Owing to the dangling bond existing on the surface of $\mathrm{MoSe}_{2}$ nanosheets, Mobased precursors are prone to adsorb on the basal plane, resulting in the formation of $\mathrm{MoS}_{2}$ quantum dots and ensuring the exposure of additional Mo-S edge sites. Without the confinement of $\mathrm{MoSe}_{2}$ nanosheets, $\mathrm{MoS}_{2}$ crystals grow freely and assemble micronized flower-like $\mathrm{MoS}_{2}$ nanosheets (Fig. S5, Supporting Information). The STEM-EDS mappings (Fig. 1f) reveal the uniform distribution of $\mathrm{Mo}$, Se, and $\mathrm{S}$ over the surface of $\mathrm{MoS}_{2} / \mathrm{MoSe}_{2^{-}}$ 0.5 heterostructures, further demonstrating the homogeneous growth of $\mathrm{MoS}_{2}$ over the $\mathrm{MoSe}_{2}$ substrate.

The XPS survey spectrum of $\mathrm{MoS}_{2} / \mathrm{MoSe}_{2}-0.5$ in Fig. $1 \mathrm{~g}$ reveals the existence of Mo, Se, and S. As illustrated in the high-resolution XPS spectra of Mo 3d, two peaks locate at 229.2 and $232.3 \mathrm{eV}$, corresponding to Mo $3 \mathrm{~d}_{5 / 2}$ and Mo $3 \mathrm{~d}_{3 / 2}$, respectively, indicating that the valence state of Mo is $+4 .^{44}$ In the meantime, the small shoulder peak at $226.4 \mathrm{eV}$, which is absent in the corresponding spectra of $\mathrm{MoSe}_{2}$ (Fig. S6a, Supporting Information), is ascribed to $\mathrm{S} 2 \mathrm{~s},{ }^{44}$ further demonstrating the existence of $\mathrm{MoS}_{2}$ species. The single doublet peak in the Se $3 \mathrm{~d}$ spectra composes of $\mathrm{Se} 3 \mathrm{~d}_{5 / 2}$ at $54.6 \mathrm{eV}$ and $\mathrm{Se} 3 \mathrm{~d}_{3 / 2}$ at $55.4 \mathrm{eV}$, while the fitted Se $3 p$ peaks consist of Se $3 p_{3 / 2}$ and $\mathrm{Se} 3 \mathrm{p}_{1 / 2}$ at 160.7 and $166.7 \mathrm{eV}$, respectively. ${ }^{45}$ For the $\mathrm{S} 2 \mathrm{p}$ spectra, the 
$\mathrm{S} 2 \mathrm{p}_{3 / 2}$ and $\mathrm{S} 2 \mathrm{p}_{1 / 2}$ peaks can be fitted at 161.8 and $164.1 \mathrm{eV}$, respectively. ${ }^{45}$ The highresolution XPS spectra of pure $\mathrm{MoS}_{2}, \mathrm{MoSe}_{2}$, and other $\mathrm{MoS}_{2} / \mathrm{MoSe}_{2}$ heterostructures can be found in Fig. S6 (Supporting Information).
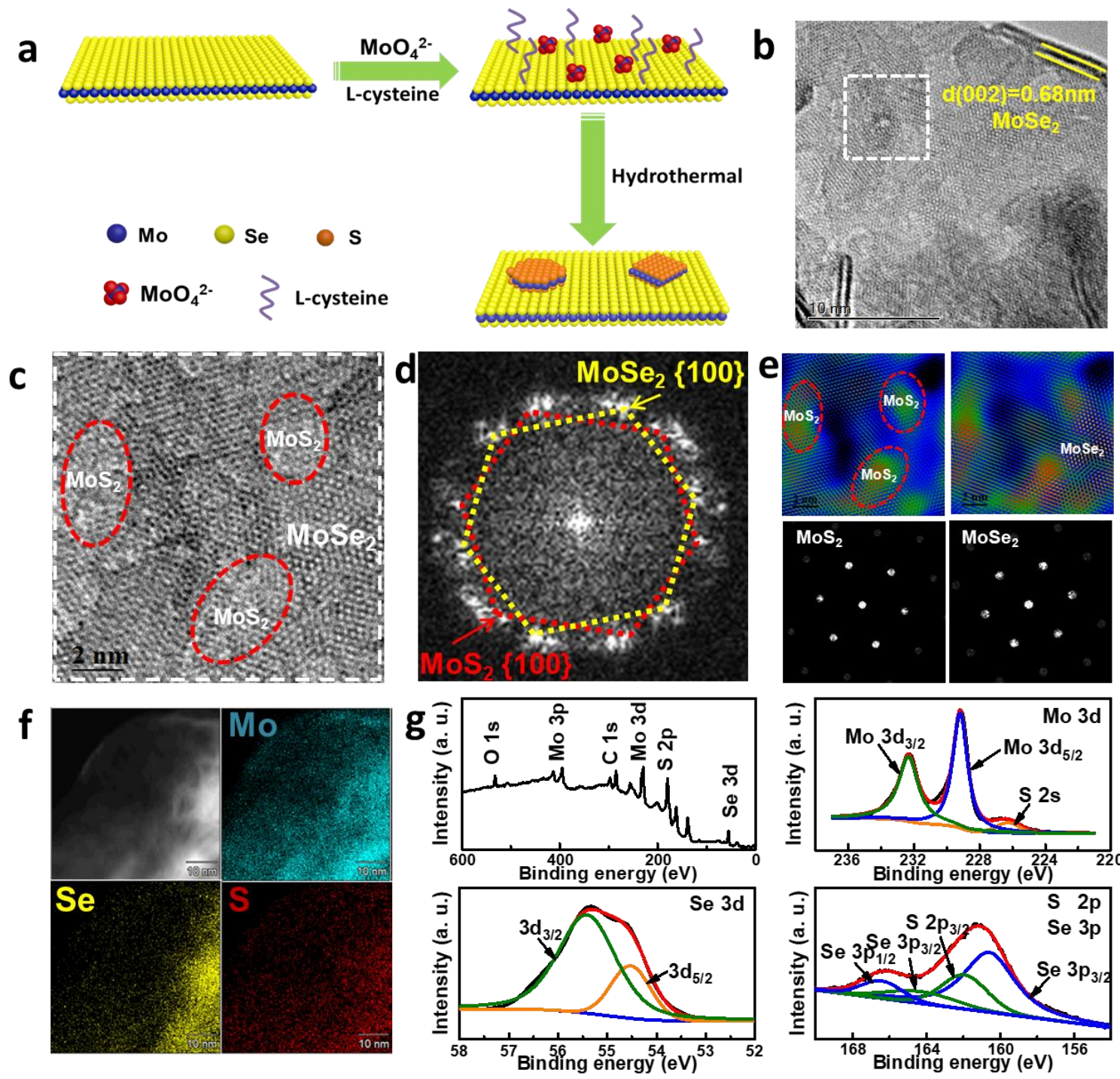

Fig. 1. (a) Schematic illustration of the synthesis of $\mathrm{MoS}_{2} / \mathrm{MoSe}_{2}$ heterostructures. (b) TEM image of $\mathrm{MoS}_{2} / \mathrm{MoSe}_{2}-0.5$ heterostructure, showing the typical nanosheets morphology. (c) HRTEM image of $\mathrm{MoS}_{2} / \mathrm{MoSe}_{2}-0.5$ heterostructure. (d) the corresponding FFT patterns of (c). (e) IFFT image generated by the selected masked FFT patterns of $\mathrm{MoSe}_{2}$ and $\mathrm{MoS}_{2}$ (top) and the selected FFT patterns (down). (f) STEM image and the corresponding EDS mapping images. (g) XPS spectra of $\mathrm{MoS}_{2} / \mathrm{MoSe}_{2}-0.5$ heterostructure. 
The electrochemical measurements were performed in $\mathrm{N}_{2}$-saturated $1.0 \mathrm{M} \mathrm{KOH}$ and $0.5 \mathrm{M}$ $\mathrm{H}_{2} \mathrm{SO}_{4}$ solution using a three-electrode setup. As shown in Fig. 2a, $\mathrm{MoS}_{2} / \mathrm{MoSe}_{2}$ heterostructures show notably enhanced performance compared to pure $\mathrm{MoSe}_{2}$ and $\mathrm{MoS}_{2}$ in alkaline solution. In particular, the $\mathrm{MoS}_{2} / \mathrm{MoSe}_{2}-0.5$ heterostructure exhibits the best HER activity with a small onset overpotential of $180 \mathrm{mV}$, which is much lower than that of pristine $\operatorname{MoSe}_{2}(270 \mathrm{mV})$ and $\mathrm{MoS}_{2}(310 \mathrm{mV})$. To achieve a cathodic current density of $10 \mathrm{~mA} \mathrm{~cm}{ }^{-2}$, the $\mathrm{MoS}_{2} / \mathrm{MoSe}_{2}-0.5$ only requires an overpotential of $235 \mathrm{mV}$ (Fig. 2b), while large overpotentials of $330 \mathrm{mV}$ and $400 \mathrm{mV}$ are required for $\mathrm{MoSe}_{2}$ and $\mathrm{MoS}_{2}$, respectively. The performance of $\mathrm{MoS}_{2} / \mathrm{MoSe}_{2}-0.5$ heterostructure is among the best of the reported carbonfree $\mathrm{MoS}_{2}$-based and $\mathrm{MoSe}_{2}$-based materials without conductive substrates under the same test condition, and even comparable to a few conductive substrate-supported catalysts (Table $\mathrm{S} 1$, Supporting Information). Meanwhile, $\mathrm{MoS}_{2} / \mathrm{MoSe}_{2}-0.5$ delivers a current density as high as $36 \mathrm{~mA} \mathrm{~cm}^{-2}$ at an overpotential of $300 \mathrm{mV}$, which is about 14 and 5 times higher than that of $\mathrm{MoS}_{2}\left(2.5 \mathrm{~mA} \mathrm{~cm}{ }^{-2}\right)$ and $\mathrm{MoSe}_{2}\left(6.5 \mathrm{~mA} \mathrm{~cm}^{-2}\right)$, respectively. Due to the relatively lower activity of $\mathrm{MoS}_{2}$, the catalytic activity of the $\mathrm{MoS}_{2} / \mathrm{MoSe}_{2}$ heterostructures decays as further increasing $\mathrm{MoS}_{2}$. The improved catalytic kinetics of $\mathrm{MoS}_{2} / \mathrm{MoSe}_{2}$ heterostructures is also evidenced by the reduced Tafel slopes. The Tafel plots of $\mathrm{MoSe}_{2}, \mathrm{MoS}_{2}$, and $\mathrm{MoS}_{2} / \mathrm{MoSe}_{2}$

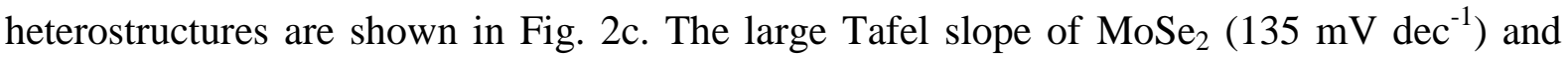
$\mathrm{MoS}_{2}\left(157 \mathrm{mV} \mathrm{dec}{ }^{-1}\right)$ reveal that the rate-determining step of HER is the water adsorption and dissociation process. Compared with bare $\mathrm{MoSe}_{2}$ and $\mathrm{MoS}_{2}$, the decreased Tafel slope of $\mathrm{MoS}_{2} / \mathrm{MoSe}_{2}-0.5$ (96 mV $\mathrm{dec}^{-1}$ ) suggests that the HER kinetics is determined by the combination of the Volmer step and the subsequent Heyrovsky step, ${ }^{46}$ indicating an accelerated water adsorption/dissociation kinetics on the $\mathrm{MoS}_{2} / \mathrm{MoSe}_{2}-0.5$ surface. Electrochemical impedance spectroscopy (EIS) is employed to further investigate the reaction process. As shown in Fig. 2d, the reaction resistance of $\mathrm{MoS}_{2} / \mathrm{MoSe}_{2}-0.5$ (30 $\Omega$ ) is smaller 
than that of either pure $\operatorname{MoSe}_{2}(98 \Omega)$ or $\mathrm{MoS}_{2}(486 \Omega)$, indicating a substantially enhanced reaction kinetics due to the combination of $\mathrm{MoS}_{2}$ quantum dots and $\mathrm{MoSe}_{2}$ nanosheets. The activity durability of the electrocatalysts was evaluated by the chronopotentiometry test as shown in Fig. 2e. It can be observed that the overpotential of $\mathrm{MoSe}_{2}$ rises distinctly within the initial $10 \mathrm{~min}$ and eventually increases by about $108 \mathrm{mV}$ during the 3 -h operation. In contrast, the overpotential of $\mathrm{MoS}_{2} / \mathrm{MoSe}_{2}-0.5$ is only elevated by $59 \mathrm{mV}$, manifesting that the stability is also improved after the incorporation of $\mathrm{MoS}_{2}$ quantum dots. The HER turnover frequencies (TOFs) were calculated to evaluate the intrinsic activity of the electrocatalysts. Here, $\mathrm{Mo}^{4+}$ was employed as the active site for calculating TOFs, and the TOFs at the overpotential of $300 \mathrm{mV}$ are shown in Fig. 2f. Remarkably, $\mathrm{MoS}_{2} / \mathrm{MoSe}_{2}-0.5$ heterostructure deliver a TOF as high as $102.3 \mathrm{~s}^{-1}$, outperforming pure $\operatorname{MoSe}_{2}\left(17.6 \mathrm{~s}^{-1}\right), \operatorname{MoS}_{2}\left(5.4 \mathrm{~s}^{-1}\right)$, $\mathrm{MoS}_{2} / \mathrm{MoSe}_{2}-0.25\left(66.7 \mathrm{~s}^{-1}\right)$ and $\mathrm{MoS}_{2} / \mathrm{MoSe}_{2}-1\left(53.7 \mathrm{~s}^{-1}\right)$.

a
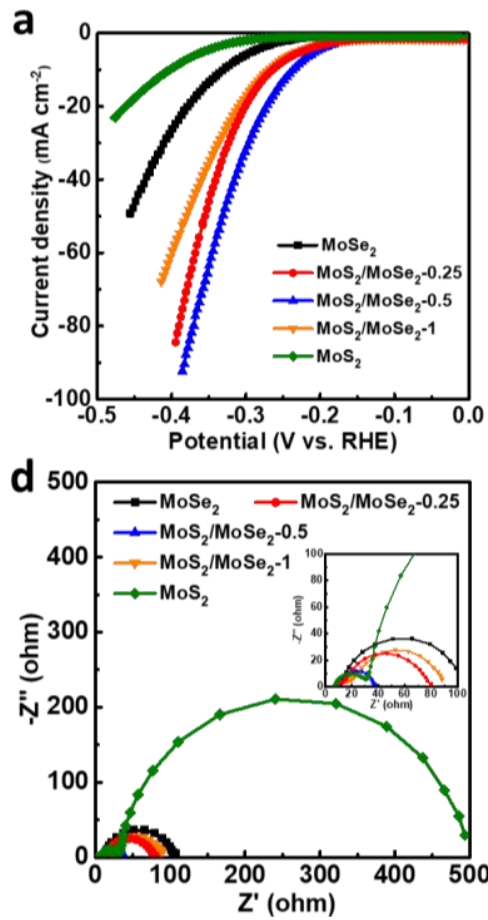
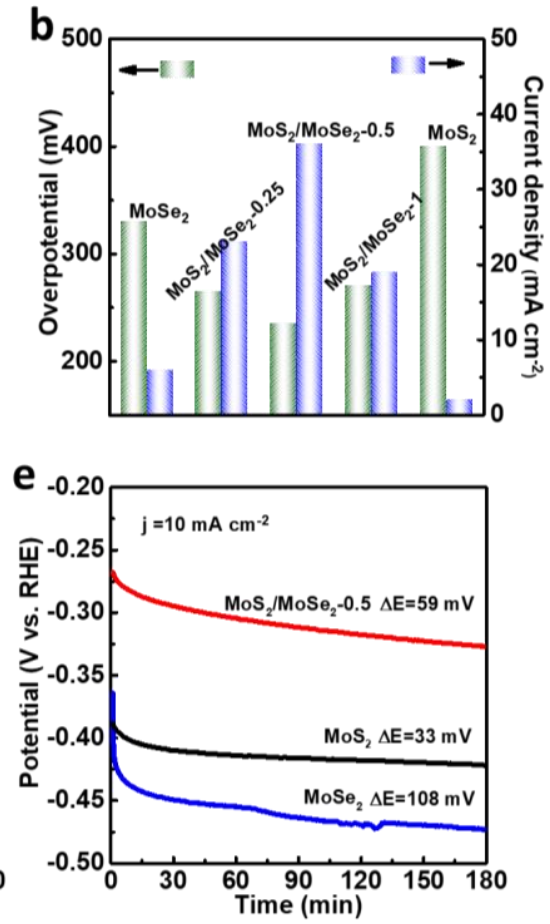
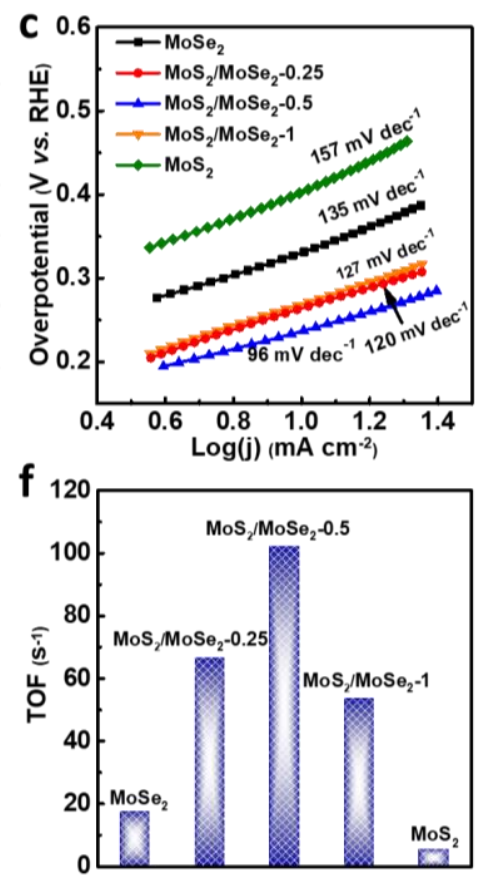

Fig. 2. (a) iR-corrected LSV curves in $1 \mathrm{M} \mathrm{KOH}$ solution of various electrocatalysts as indicated (scan rate: $5 \mathrm{mV} \mathrm{s}^{-1}$, catalyst mass loading: $0.204 \mathrm{mg} \mathrm{cm}^{-2}$ ). (b) Overpotential 
required at $10 \mathrm{~mA} \mathrm{~cm}^{-2}$ and current densities at an overpotential of $300 \mathrm{mV}$; (c) Tafel plots derived from LSV curves, (d) Nyquist plot measured at an overpotential of $300 \mathrm{mV}$; (e) chronopotentiometry curves conducted at a constant current density of $10 \mathrm{~mA} \mathrm{~cm}$; (f) TOF of $\mathrm{MoSe}_{2}, \mathrm{MoS}_{2}$ and $\mathrm{MoS}_{2} / \mathrm{MoSe}_{2}$ heterostructures at the overpotential of $0.3 \mathrm{~V}$.

To investigate the origination of the promoted catalytic activity of $\mathrm{MoSe}_{2}$, the acidic HER performance evaluation was also carried out as shown in Fig. 3a. Interestingly, $\mathrm{MoSe}_{2}$ exhibits the lowest overpotential $(205 \mathrm{mV})$ at the current density of $10 \mathrm{~mA} \mathrm{~cm}$, and the electrocatalytic activity of $\mathrm{MoS}_{2} / \mathrm{MoSe}_{2}$ heterostructures is not improved. The catalytic activity variation at high current density region can be ascribed to the different hydrogen desorption behaviour at high hydrogen coverage. ${ }^{47,48}$ To obtain deeper insight into the electrochemical behaviour, the electrochemical active surface areas (ECSA) of the catalysts were estimated through evaluating the electrochemical double layer capacitance $\left(\mathrm{C}_{\mathrm{dl}}\right)$ in a non-Faraday region in alkaline as well as acidic solutions (Fig. S7, Supporting Information). As shown in Fig. 3b, $\mathrm{MoS}_{2} / \mathrm{MoSe}_{2}-0.5$ and pure $\mathrm{MoSe}_{2}$ possess similar $\mathrm{C}_{\mathrm{dl}}$ in both alkaline and acidic solutions, demonstrating similar ECSA of $\mathrm{MoS}_{2} / \mathrm{MoSe}_{2}-0.5$ and pure $\mathrm{MoSe}_{2}$. Therefore, the promoted alkaline HER activity can be ascribed to the accelerated water adsorption/dissociation process that originates from the additional edge sites in these molybdenum dichalcogenides. 

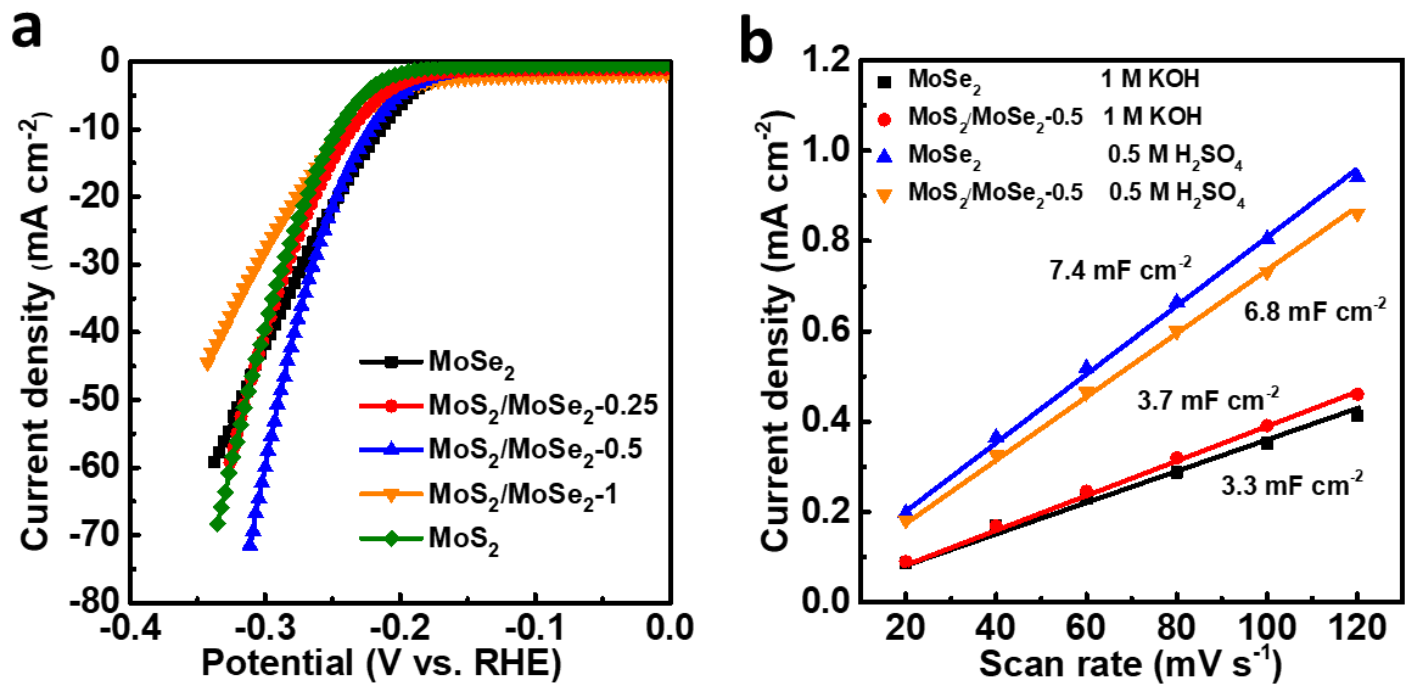

Fig. 3. (a) iR-corrected LSV curves measured at a scan rate of $5 \mathrm{mV} \mathrm{s}^{-1}$ in $0.5 \mathrm{M} \mathrm{H}_{2} \mathrm{SO}_{4}$ solution; (b) Fitted $\mathrm{C}_{\mathrm{dl}}$ of $\mathrm{MoSe}_{2}$ and $\mathrm{MoS}_{2} / \mathrm{MoSe}_{2}-0.5$ at $0 \mathrm{~V}$ vs. RHE (1 M KOH) and 0.05 V vs. $\mathrm{RHE}\left(0.5 \mathrm{M} \mathrm{H}_{2} \mathrm{SO}_{4}\right)$.

To further verify the feasibility of engineering additional edge sites for promoting alkaline HER, $\mathrm{MoSe}_{2} / \mathrm{MoSe}_{2}-0.5$ and $\mathrm{MoS}_{2} / \mathrm{MoS}_{2}-0.5$ heterostructures were synthesized. As illustrated in Fig. S8 (Supporting Information), $\mathrm{MoSe}_{2} / \mathrm{MoSe}_{2}-0.5$ and $\mathrm{MoS}_{2} / \mathrm{MoS}_{2}-0.5$ possess the same diffraction peaks with pristine $\mathrm{MoSe}_{2}$ and $\mathrm{MoS}_{2}$, respectively. Both $\mathrm{MoSe}_{2} / \mathrm{MoSe}_{2}-0.5$ and $\mathrm{MoS}_{2} / \mathrm{MoS}_{2}-0.5$ maintain the graphene-like nanosheet morphology (Fig. S9 and S10, Supporting Information). $\mathrm{MoSe}_{2}$ and $\mathrm{MoS}_{2}$ quantum dots can be identified from the heterostructures in HRTEM images (Fig. S11a and c, Supporting Information). Also, the relative intensity variation in the selected area further demonstrates the presence of $\mathrm{MoSe}_{2}$ and $\mathrm{MoS}_{2}$ quantum dots on $\mathrm{MoSe}_{2}$ nanosheets and $\mathrm{MoS}_{2}$ nanosheets, respectively (Fig. S11b and d, Supporting Information). The LSV curves of $\mathrm{MoSe}_{2} / \mathrm{MoSe}_{2}-0.5$ and $\mathrm{MoS}_{2} / \mathrm{MoS}_{2}-0.5$ in $1 \mathrm{M} \mathrm{KOH}$ and $0.5 \mathrm{M} \mathrm{H}_{2} \mathrm{SO}_{4}$ were shown in Fig. $4 \mathrm{a}$ and b. Similar to $\mathrm{MoS}_{2} / \mathrm{MoSe}_{2}$ heterostructures, the overpotential to reach a current density of $10 \mathrm{~mA} \mathrm{~cm}{ }^{-2}$ of both $\mathrm{MoSe}_{2} / \mathrm{MoSe}_{2}-0.5(250 \mathrm{mV})$ and $\mathrm{MoS}_{2} / \mathrm{MoS}_{2}-0.5(331 \mathrm{mV})$ are substantially decreased as compared with $\mathrm{MoSe}_{2}(330 \mathrm{mV})$ and $\mathrm{MoS}_{2}(400 \mathrm{mV})$ in $1 \mathrm{M} \mathrm{KOH}$ solution, in sharp 
contrast with the negligible overpotential decrease in acidic solution. The reduction of the Tafel slopes (Fig. 4c and d) also verify the enhanced electrochemical reaction kinetics of the heterostrutures. Furthermore, we prepared two control samples: pure $\mathrm{MoSe}_{2}$ nanosheets treated with a second hydrothermal process in DI water at $180{ }^{\circ} \mathrm{C}$ for $24 \mathrm{~h}$ (denoted as $\mathrm{MoSe}_{2}-2$ ) and a physical mixture of $\mathrm{MoSe}_{2}$ and $\mathrm{MoS}_{2}$ nanosheets (denoted as $\mathrm{MoS}_{2} / \mathrm{MoSe}_{2}-$ m). As shown in Fig. S12 (Supporting Information), $\mathrm{MoSe}_{2}-2$ exhibits almost the same electrocatalytic activity with pristine $\mathrm{MoSe}_{2}$, proving that the HER performance is not affected by the additional hydrothermal treatment. On the other hand, the catalytic activity of $\mathrm{MoS}_{2} / \mathrm{MoSe}_{2}-\mathrm{m}$ is between pure $\mathrm{MoS}_{2}$ and $\mathrm{MoSe}_{2}$, confirming the significant role that the extra edge sites play in the enhanced HER activity in alkaline solution.
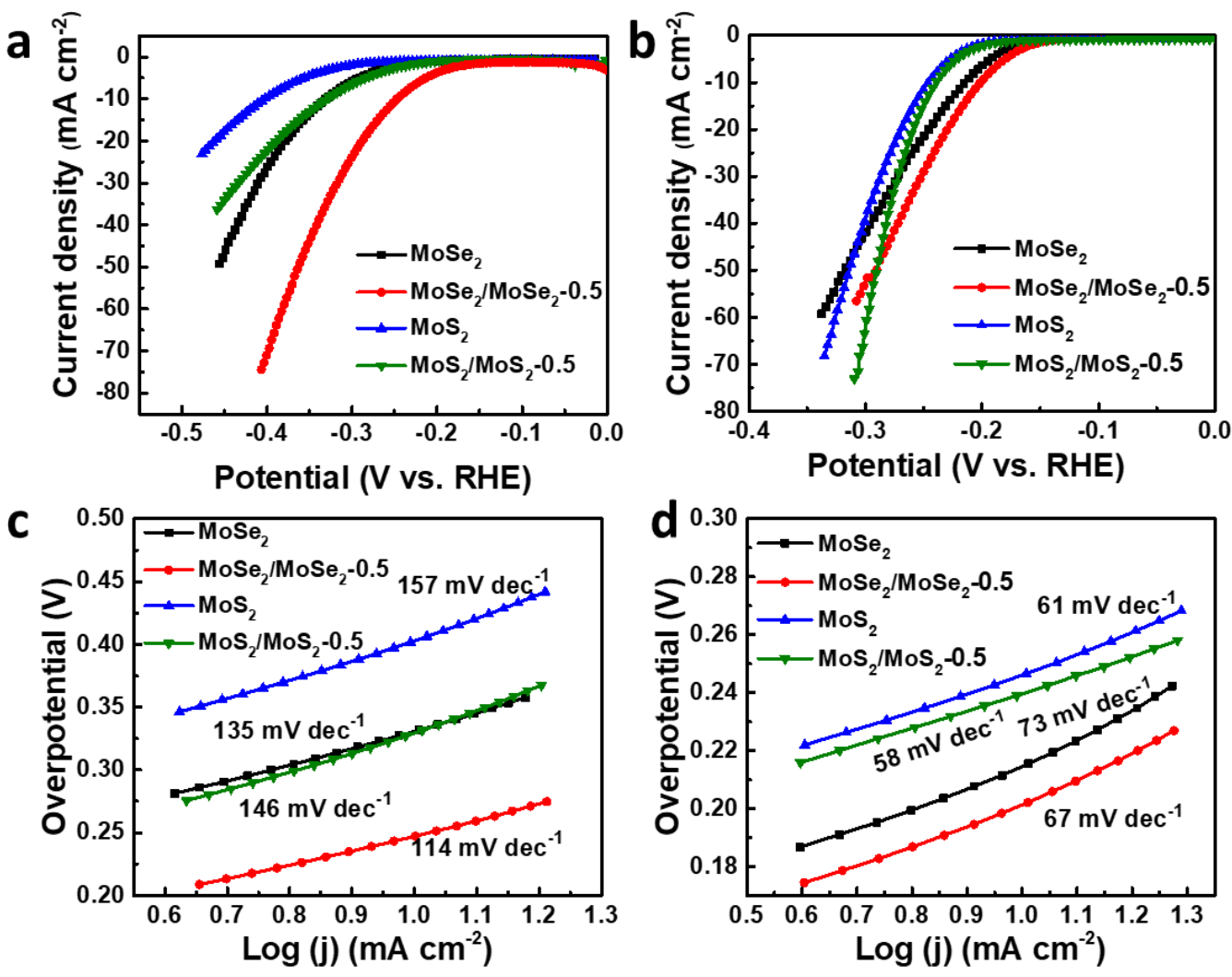

Fig. 4. iR-corrected LSV curves of $\mathrm{MoSe}_{2}, \mathrm{MoSe}_{2}, \mathrm{MoSe}_{2} / \mathrm{MoSe}_{2}-0.5$, and $\mathrm{MoS}_{2} / \mathrm{MoS}_{2}-0.5$ measured at a scan rate of $5 \mathrm{mV} \mathrm{s}^{-1}$ in (a) $1 \mathrm{M} \mathrm{KOH}$ solution and (b) $0.5 \mathrm{M} \mathrm{H}_{2} \mathrm{SO}_{4}$ solution; Tafel plots derived from LSV curves in (c) $1 \mathrm{M} \mathrm{KOH}$ solution and (d) $0.5 \mathrm{M} \mathrm{H}_{2} \mathrm{SO}_{4}$ solution. 
As has been reported, the adsorption/dissociation of $\mathrm{H}_{2} \mathrm{O}$ is more favoured on the Mo-edges rather than the basal plane of molybdenum dichalcogenides. ${ }^{40,49,50}$ Thus, the improved alkaline HER performance for the TMDs-based heterostructures is derived from the additional edge sites provided by $\mathrm{MoS}_{2}$ or $\mathrm{MoSe}_{2}$ nanoclusters. As shown in Fig. 5, Mo-edge sites in $\mathrm{MoS}_{2}$ act as a promoter for water adsorption and dissociation, while the produced hydrogen intermediates absorb on nearby $\mathrm{MoSe}_{2}$ and subsequently combined to hydrogen gas. In this way, $\mathrm{MoS}_{2} / \mathrm{MoSe}_{2}$ heterostructure effectively reduces the energy barrier of the water dissociation and accelerates the alkaline HER process accordingly.

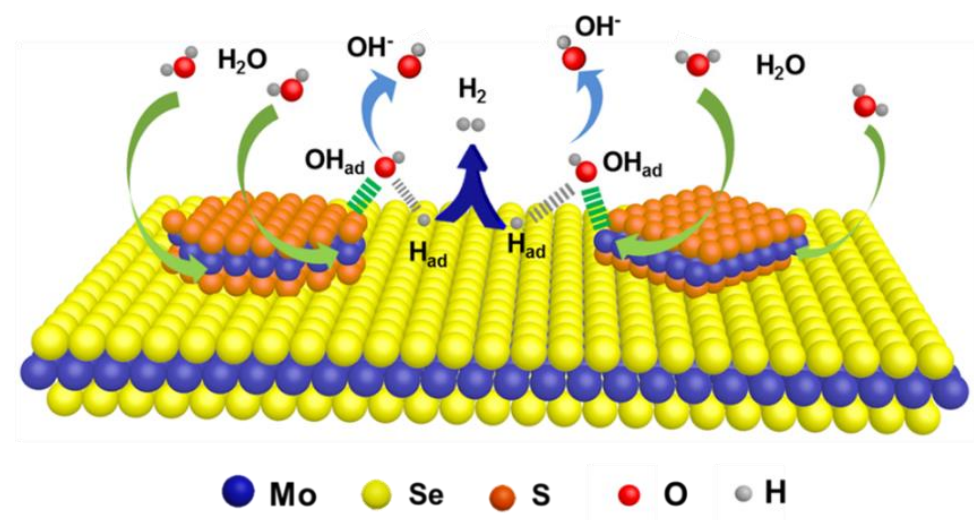

Fig. 5. HER mechanism on $\mathrm{MoS}_{2} / \mathrm{MoSe}_{2}$ heterostructure in alkaline solution.

\section{Conclusion}

In summary, TMDs-based heterostructures $\left(\mathrm{MoS}_{2} / \mathrm{MoSe}_{2}, \mathrm{MoSe}_{2} / \mathrm{MoSe}_{2}\right.$ and $\left.\mathrm{MoS}_{2} / \mathrm{MoS}_{2}\right)$ with $\mathrm{MoS}_{2}$ or $\mathrm{MoSe}_{2}$ quantum dots anchored on TMDs nanosheets were synthesized via a two-step chemical solution process. The TMDs-based heterostructures delivered substantially improved catalytic activity over their single-component counterparts in alkaline media. The abundant edge sites in $\mathrm{MoS}_{2}$ or $\mathrm{MoSe}_{2}$ quantum dots would enhance the $\mathrm{H}_{2} \mathrm{O}$ affinity of the TMDs-based heterostructures and hence promote the water adsorption/dissociation capability and alkaline HER kinetics accordingly. We believe the strategy for creating extra edge sites 
by engineering hetero-nanostructures would provide new insights into designing efficient electrocatalysts for hydrogen evolution in alkaline media.

\section{Acknowledgements}

This work was financially supported by the Australian Research Council (ARC) DECRA Grant (DE160100596), ARC Discovery Project (DP160102627), and AIIM FOR GOLD Grant $(2017,2018)$. The authors also acknowledge the use of facilities within the UOW Electron Microscopy Centre.

\section{References}

1. T. E. Mallouk, Nat. chem., 2013, 5, 362.

2. H. I. Karunadasa, E. Montalvo, Y. Sun, M. Majda, J. R. Long and C. J. Chang, Science, 2012, 335, 698-702.

3. J. Mei, Y. Zhang, T. Liao, Z. Sun and S. X. Dou, Natl. Sci. Rev., 2017, 5, 389-416.

4. G. Zhao, K. Rui, S. X. Dou and W. Sun, Adv. Funct. Mater., 2018, DOI: doi:10.1002/adfm.201803291, 1803291.

5. G. W. Crabtree, M. S. Dresselhaus and M. V. Buchanan, Phys. Today, 2004, 57, 39-44.

6. R. F. Service, Science (New York, NY), 2009, 324, 1257.

7. X. Zhang and Y. Liang, Adv. Sci., 2018, 5, 1700644.

8. G. F. Chen, T. Y. Ma, Z. Q. Liu, N. Li, Y. Z. Su, K. Davey and S. Z. Qiao, Adv. Funct. Mater., 2016, 26, 3314-3323.

9. J. Wang, L. Gan, W. Zhang, Y. Peng, H. Yu, Q. Yan, X. Xia and X. Wang, Sci. Adv., 2018, 4, eaap7970.

10. Z. Dai, H. Geng, J. Wang, Y. Luo, B. Li, Y. Zong, J. Yang, Y. Guo, Y. Zheng and X. Wang, ACS Nano, 2017, 11, 11031-11040.

11. H. Fan, H. Yu, Y. Zhang, Y. Zheng, Y. Luo, Z. Dai, B. Li, Y. Zong and Q. Yan, Angew. Chem. Int. Ed., 2017, 56, 12566-12570.

12. Y. Chen, K. Rui, J. Zhu, S. X. Dou and W. Sun, Chem.- Eur. J., 2018, DOI: doi.org/10.1002/chem.201802068.

13. Y. Chen, Q. Zhou, G. Zhao, Z. Yu, X. Wang, S. X. Dou and W. Sun, Adv. Funct. Mater., 2018, 28, 1705583.

14. S. H. Ye, Z. X. Shi, J. X. Feng, Y. X. Tong and G. R. Li, Angew. Chem. Int. Ed., 2018, 57, 2672-2676.

15. J. X. Feng, S. H. Ye, H. Xu, Y. X. Tong and G. R. Li, Adv. Mater., 2016, 28, 4698-4703.

16. Q. Gong, L. Cheng, C. Liu, M. Zhang, Q. Feng, H. Ye, M. Zeng, L. Xie, Z. Liu and Y. Li, ACS Catal., 2015, 5, 2213-2219.

17. H. Li, C. Tsai, A. L. Koh, L. Cai, A. W. Contryman, A. H. Fragapane, J. Zhao, H. S. Han, H. C. Manoharan and F. Abild-Pedersen, Nat. Mater., 2016, 15, 48.

18. J. Duan, S. Chen, B. A. Chambers, G. G. Andersson and S. Z. Qiao, Adv. Mater., 2015, 27, 4234-4241.

19. G. Zhao, P. Li, K. Rui, Y. Chen, S. X. Dou and W. Sun, Chem.- Eur. J., 2018, DOI: 10.1002/chem.201801693.

20. S. Feng, X. Li, J. Huo, Q. Li, C. Xie, T. Liu, Z. Liu, Z. Wu and S. Wang, ChemCatChem, 2018, 10, 796-803. 
21. C. Ray, S. C. Lee, B. Jin, A. Kundu, J. H. Park and S. C. Jun, J. Mater. Chem. A, 2018, 6, 44664476.

22. Q. Chen, R. Wang, M. Yu, Y. Zeng, F. Lu, X. Kuang and X. Lu, Electrochimica Acta, 2017, 247, 666-673.

23. H. Lin, Z. Shi, S. He, X. Yu, S. Wang, Q. Gao and Y. Tang, Chem. Sci., 2016, 7, 3399-3405.

24. C. Wan, Y. N. Regmi and B. M. Leonard, Angew. Chem., 2014, 126, 6525-6528.

25. F. H. Saadi, A. I. Carim, E. Verlage, J. C. Hemminger, N. S. Lewis and M. P. Soriaga, J. Phys. Chem. C, 2014, 118, 29294-29300.

26. A.-L. Wang, J. Lin, H. Xu, Y.-X. Tong and G.-R. Li, J. Mater. Chem. A, 2016, 4, 16992-6999.

27. J.-X. Feng, S.-Y. Tong, Y.-X. Tong and G.-R. Li, J. Am. Chem. Soc., 2018, 140, 5118-5126.

28. Q. Liang, L. Zhong, C. Du, Y. Zheng, Y. Luo, J. Xu, S. Li and Q. Yan, Adv. Funct. Mater., 2018, 1805075.

29. L. Xie, X. Ren, Q. Liu, G. Cui, R. Ge, A. M. Asiri, X. Sun, Q. Zhang and L. Chen, J. Mater. Chem. A, 2018, 6, 1967-1970.

30. L. Wang, C. Lin, D. Huang, J. Chen, L. Jiang, M. Wang, L. Chi, L. Shi and J. Jin, ACS Catal., 2015, 5, 3801-3806.

31. G. Zhao, Y. Lin, K. Rui, Q. Zhou, Y. Chen, S. Dou and W. Sun, Nanoscale, 2018.

32. F. Lai, D. Yong, X. Ning, B. Pan, Y. E. Miao and T. Liu, Small, 2017, 13, 1602866.

33. X. Geng, W. Wu, N. Li, W. Sun, J. Armstrong, A. Al- hilo, M. Brozak, J. Cui and T. P. Chen, Adv. Funct. Mater., 2014, 24, 6123-6129.

34. T. F. Jaramillo, K. P. Jørgensen, J. Bonde, J. H. Nielsen, S. Horch and I. Chorkendorff, Science, 2007, 317, 100-102.

35. Q. Ding, B. Song, P. Xu and S. Jin, Chem., 2016, 1, 699-726.

36. Z. F. Huang, J. Song, K. Li, M. Tahir, Y. T. Wang, L. Pan, L. Wang, X. Zhang and J. J. Zou, J. Am. Chem. Soc., 2016, 138, 1359-1365.

37. Y. Liu, J. Wu, K. P. Hackenberg, J. Zhang, Y. M. Wang, Y. Yang, K. Keyshar, J. Gu, T. Ogitsu and R. Vajtai, arXiv preprint arXiv:1608.05755, 2016.

38. B. Hinnemann, P. G. Moses, J. Bonde, K. P. Jørgensen, J. H. Nielsen, S. Horch, I. Chorkendorff and J. K. Nørskov, J. Am. Chem. Soc., 2005, 127, 5308-5309.

39. D. Kong, H. Wang, J. J. Cha, M. Pasta, K. J. Koski, J. Yao and Y. Cui, Nano Lett., 2013, 13, 1341-1347.

40. K. K. Ghuman, S. Yadav and C. V. Singh, J. Phys. Chem. C, 2015, 119, 6518-6529.

41. Q. Zhou, Y. Chen, G. Zhao, Y. Lin, Z. Yu, X. Xu, X. Wang, H. K. Liu, W. Sun and S. X. Dou, ACS Catal., 2018, 8, 5382-5390.

42. H. Li, C. Tsai, A. L. Koh, L. Cai, A. W. Contryman, A. H. Fragapane, J. Zhao, H. S. Han, H. C. Manoharan and F. Abild-Pedersen, Nat. Mater., 2016, 15, 48.

43. W. Jung, S. Lee, D. Yoo, S. Jeong, P. Miró, A. Kuc, T. Heine and J. Cheon, J. Am. Chem. Soc., 2015, 137, 7266-7269.

44. Y. Luo, X. Li, X. Cai, X. Zou, F. Kang, H.-M. Cheng and B. Liu, ACS Nano, 2018, 12, 45654573.

45. J. Yang, J. Zhu, J. Xu, C. Zhang and T. Liu, ACS Appl. Mater. Inter., 2017, 9, 44550-44559.

46. Y. Li, H. Wang, L. Xie, Y. Liang, G. Hong and H. Dai, J. Am. Chem. Soc., 2011, 133, 72967299.

47. E. Skúlason, G. S. Karlberg, J. Rossmeisl, T. Bligaard, J. Greeley, H. Jónsson and J. K. Nørskov, Phys. Chem. Chem. Phys., 2007, 9, 3241-3250.

48. A. R. Kucernak and C. Zalitis, J. Phys. Chem. C, 2016, 120, 10721-10745.

49. C. Ataca and S. Ciraci, Phys. Rev. B, 2012, 85, 195410.

50. B. Zhang, J. Liu, J. Wang, Y. Ruan, X. Ji, K. Xu, C. Chen, H. Wan, L. Miao and J. Jiang, Nano Energy, 2017, 37, 74-80. 


\section{Figure captions}

Figure 1 (a) Schematic illustration of the synthesis of $\mathrm{MoS}_{2} / \mathrm{MoSe}_{2}$ heterostructures. (b) TEM image of $\mathrm{MoS}_{2} / \mathrm{MoSe}_{2}-0.5$ heterostructure, showing the typical nanosheets morphology. (c) HRTEM image of $\mathrm{MoS}_{2} / \mathrm{MoSe}_{2}-0.5$ heterostructure. (d) the corresponding FFT patterns of (c). (e) IFFT image generated by the selected masked FFT patterns of $\mathrm{MoSe}_{2}$ and $\mathrm{MoS}_{2}$ (top) and the selected FFT patterns (down). (f) STEM image and the corresponding EDS mapping images. (g) XPS spectra of $\mathrm{MoS}_{2} / \mathrm{MoSe}_{2}-0.5$ heterostructure.

Figure 2 (a) iR-corrected LSV curves in $1 \mathrm{M} \mathrm{KOH}$ solution of various electrocatalysts as indicated (scan rate: $5 \mathrm{mV} \mathrm{s}^{-1}$, catalyst mass loading: $0.204 \mathrm{mg} \mathrm{cm}^{-2}$ ). (b) Overpotential required at $10 \mathrm{~mA} \mathrm{~cm}^{-2}$ and current densities at an overpotential of $300 \mathrm{mV}$; (c) Tafel plots derived from LSV curves, (d) Nyquist plot measured at an overpotential of $300 \mathrm{mV}$; (e) chronopotentiometry curves conducted at a constant current density of $10 \mathrm{~mA} \mathrm{~cm}{ }^{-2}$; (f) TOF of $\mathrm{MoSe}_{2}, \mathrm{MoS}_{2}$ and $\mathrm{MoS}_{2} / \mathrm{MoSe}_{2}$ heterostructures at the overpotential of $0.3 \mathrm{~V}$.

Figure 3 (a) iR-corrected LSV curves measured at a scan rate of $5 \mathrm{mV} \mathrm{s}^{-1}$ in $0.5 \mathrm{M} \mathrm{H}_{2} \mathrm{SO}_{4}$ solution; (b) Fitted $\mathrm{C}_{\mathrm{dl}}$ of $\mathrm{MoSe}_{2}$ and $\mathrm{MoS}_{2} / \mathrm{MoSe}_{2}-0.5$ at $0 \mathrm{~V}$ vs. RHE (1 M KOH) and 0.05 V vs. RHE (0.5 $\left.\mathrm{M} \mathrm{H}_{2} \mathrm{SO}_{4}\right)$.

Fig. 4. iR-corrected LSV curves of $\mathrm{MoSe}_{2}, \mathrm{MoSe}_{2}, \mathrm{MoSe}_{2} / \mathrm{MoSe}_{2}-0.5$, and $\mathrm{MoS}_{2} / \mathrm{MoS}_{2}-0.5$ measured at a scan rate of $5 \mathrm{mV} \mathrm{s}^{-1}$ in (a) $1 \mathrm{M} \mathrm{KOH}$ solution and (b) $0.5 \mathrm{M} \mathrm{H}_{2} \mathrm{SO}_{4}$ solution; Tafel plots derived from LSV curves in (c) $1 \mathrm{M} \mathrm{KOH}$ solution and (d) $0.5 \mathrm{M} \mathrm{H}_{2} \mathrm{SO}_{4}$ solution.

Figure 5 HER mechanism on $\mathrm{MoS}_{2} / \mathrm{MoSe}_{2}$ heterostructure in alkaline solution. 